\title{
Improving Influenza Vaccination Rates- A Straightforward Task or a Multifaceted Challenge?
}

\author{
Ljiljana Trtica-Majnaric ${ }^{1,2}$ \\ ${ }^{1}$ Department of Family Medicine, School of Medicine, JJ Strossmayer University of Osijek, Osijek, Croatia; ${ }^{2}$ Department of Internal \\ Medicine, School of Medicine, JJ Strossmayer University of Osijek, Osijek, Croatia. \\ Email: ljiljana.majnaric@hi.t-com.hr
}

Received March 30 $0^{\text {th }}, 2013$; revised April 30 $0^{\text {th }}, 2013$; accepted May $8^{\text {th }}, 2013$

Copyright (C) 2013 Ljiljana Trtica-Majnaric. This is an open access article distributed under the Creative Commons Attribution License, which permits unrestricted use, distribution, and reproduction in any medium, provided the original work is properly cited.

\begin{abstract}
Annual vaccination with trivalent inactivated vaccines has been proven as safe and efficacious in preventing influenza and its complications. It is recommended especially to the elderly $(>65)$ and other people at high risk for influenza complications and death such as patients with chronic medical conditions. Healthcare workers, who are considered to transmit infection to patients, or reciprocally, can be infected during encounters with patients, are also strongly advised to regularly receive vaccines. In order to improve influenza vaccination rates in countries in Europe, health authorities set targets for vaccination coverage by 2010. Despite the substantial efforts done, coverage rates maintain low. It is considered that informed decisions, based on existing evidence, are likely to cope with improving vaccination rates. Intention of this manuscript is to address some important issues connected with influenza vaccination which, to be able to aid the evidence, need to be further clearified. To support the debate, the author presented some dubious facts from the own practice experiences. As a long-lasting solution to improve vaccination practice strategies, strengthening programed vaccination is suggested. This concept would include implementation of nationwide vaccination protocols and their harmonization by the common logistics, and standardized data collection based on installation of E-health records. This strategy would allow data comparison among different populations. As based on this debate, improving influenza vaccination rates is not likely to be easy to perform straightforward task, but a multifaceted, long term challenge.
\end{abstract}

Keywords: Influenza; Vaccination Rates; Barriers; Challenges

\section{Influenza Vaccination between Recommendations and Practice}

Each season influenza epidemics impose extra costs on health care systems and economies of the countries throughout the world [1,2]. Particularly at high risk of hospitalization and death are elderly people $(>65)$. This is thought to be a result of the aging process, characterized with a decrease in immune functions (immunosenescence) and an increase in comorbid disorders [3,4]. Fortunately, influenza is one of the most preventative disease by means of vaccination. Trivalent inactivated vaccines have been used for decades and have proven safe and efficacious in preventing disease and its complications $[5,6]$. Annual vaccination is recommended to the elderly and other people at increased risk for influenza complications, such as patients with chronic medical conditions regardless of age, residents of long-term care facilities, pregnant women and small children 6 months to 5 years old [7]. People who usually transmit influenza to those at high risk, including caregivers, household contacts and healthcare workers, are also strongly advised to regularly take vaccines [7]. Thus, current vaccination policy is predominantly self-protected. Only indirectly, when a substantial part of population attain specific immunity, the chain of infection transmission can be disrupted (herd immunity), resulting in a limitation of epidemic growth [8]. For this reason, initiatives are emerging, in some countries, to give vaccines to healthy pre-school and school children, because they are the most responsible for rapid infection spreading, through the contacts in the local communities [9].

Despite the fact that safe vaccine with proven efficacy is widely available, vaccination rates in high risk groups maintain low. A number of papers recently published in this area are dealing with the problem of how to increase vaccination uptake $[10,11]$. This demand has been em- 
phasized in European countries especially in recent years, due to the reasons such as the increasing size of the older population and the pandemic threat. In order to establish influenza vaccination as part of a comprehensive healthy aging strategy, health authorities set targets for adults 60 years of age or older of $50 \%$ vaccination coverage by 2006 and $75 \%$ by 2010 [12]. The majority of European countries to date have not achieved these proclaimed vaccination rates [13]. The question addressed here is, why is it, despite much efforts done, so hardly achieving aim?

\section{Controversy on Influenza Vaccine Effectiveness}

Controversy arises from the fact that influenza vaccination is primarily advised to subjects from high risk groups, who, on the other hand, are likely to have impaired immune response. That means that influenza vaccine is less effective in these groups, than in young, healthy adults, who otherwise are not proposed for regular vaccination. For illustration, in elderly population, percentage of subjects who after influenza vaccination achieve protective antibody levels is in general $40 \%$ $60 \%$, in comparison to $70 \%-90 \%$, in healthy middleaged adults [14]. Another disparity, in regard to influenza vaccination of older people, is a difference in vaccine efficacy among particular vaccine components, with the component $\mathrm{A} / \mathrm{H} 1 \mathrm{~N} 1$ showing substantially lower protection rates, in comparison to the other two, $\mathrm{A} / \mathrm{H} 3 \mathrm{~N} 2$ and $\mathrm{B}$. This is considered to be a result of differences in a primary antigenic exposure, the phenomenon called an original antigenic sin [15].

Another controversy is associated with the inconsistency of the reports on immune responses to influenza vaccination in high risk groups. In this regard, there are reports indicating the same effectiveness of influenza vaccination in high risk groups, as in young control groups [16]. Some other reports show that even within the same high risk group, some individuals are capable of eliciting a protective immune response, while others are not [6]. Finally, a considerable heterogeneity due to immune responsiveness exists within the elderly population group. In terms of that, clear benefits from influenza vaccination of healthy elderly people, in contrast to those with chronic medical conditions, have not been proved [17].

One more uncertainty arises on the question whether is there a posibility, in elderly persons previously vaccinated for many times, for vaccine efficacy to decrease? This possibilty is based on observations that, after repeated vaccinations, some immune mechanisms such as previous antigenic exposures, cross-reactivity, or lower antibody affinity, can interfere with the vaccine efficacy [18]. Nevertheles, apart from the immune measures of efficacy, studies show that in older, repeatedly vaccinated people, clinical outcomes are likely to be beneficial (in terms of increasing longevity and decreasing mortality), even over the course of ten seasons of successive vaccination [19]. These facts should be proved in future research and more clearly highlighted in vaccination campaigns.

\section{Factors Affecting Immune Response after Influenza Vaccination}

As mentioned above, there is a substantial heterogeneity in immune responses after influenza vaccination, beyond the selection of people into high risk or low risk groups for influenza complications. It imposes on health care providers a need for a more personalized approach in detecting persons who will likely to poorly respond to influenza vaccine. As known so far, multiple factors, including older age, chronic diseases and past exposure to influenza viruses (original antigenic sin, the number of previous vaccinations and preexisting antibody titres) can all affect variability of immune response to influenza vaccination [20]. In regard to chronic diseases, stages of a disease development, co-morbid conditions, or just biochemical subclinical disorders, may all contribute to the differences of the immune response [21]. Understanding of how these multiple factors work together is possible only by using mathematical models and computer-based methods $[22,23]$. Computer simulations based on using these methods should complement (and precede) observational studies [24].

\section{Correlates of Protection and Novel Vaccination Strategies}

Conventional vaccination approach against seasonal influenza is a trivalent inactivated split vaccine [15]. Vaccine composition is revised annually, in order to match the most prevalent circulating strains [25]. In a case of pandemic, as events from recent past have learned us, new pandemic strains may be added to the vaccination schedule [26].

Since influenza vaccine is composed of fragments containing mainly surface antigens, hemagglutinin and neuraminidase, it predominantly initiates humoral immune response [27]. Thus, the ability of influenza vaccine to provide protection against infection, expressed as an immune correlate of protection, is based on measuring specific antibody production [28]. The problem is that several measures are available and non-uniformly used in clinical studies [3]. Another problem is non concordance between the vaccine efficacy (refers to ideal conditions of randomised controlled trials) and effectiveness (refers to ordinary field conditions of public health programs and depends on the conditions under which the vaccine is 
applied in a real life) [29]. Thus, to establish more rational background for vaccination recommendations, the challenge will be standardisation of measures of protection and development of the index scale, according to which the effectiveness can be adjusted for confounding factors in different populations, such as age, health-status, region, socio-economic status, etc [30].

Current influenza vaccines are designed primarily to protect immunized individuals from the occurence of clinical disease, according to the neutralizing properties of specific antibodies which production is initiated after vaccination [31]. However, in providing protection against influenza related complications such as pneumonia, specific cell-mediated immunity has been proven to be more important [32]. Recent findings in experimental immunology highlight the importance of the early signals mediated by the non-specific immune system, for the development of the immune response [33]. Since postvaccination antibody production significantly varies in elderly population, there are initiatives to establish other immunologic parameters as correlates of protection [34]. To date, well validated immunologic markers, applicable for practical use, have not been identified [35]. This may be due to the extreme heterogeneity of the immune disorders in elderly population and the fact that the best post-vaccination protection is achieved when all arms of the immune system are preserved and coordinately act together [36,37]. For this reason, the challenge for the future will be careful selection of older people for participation in the immunologic studies, by taking into account their comorbid disorders. This will likely to allow greater understanding the age-related changes and chronic comorbid conditions and their connections with immune disorders. Some new integrative approaches, such as a systems biology, and new technologies, such as reverse genetics, are likely to improve our understanding of these issues $[38,39]$.

In order to improve influenza vaccination efficacy in the elderly and other high risk groups, new vaccines and vaccination approaches are now being pursued [40]. Advances in the correlates of immune protection may aid their development [35]. Also important, to improve vaccination efficacy, would be stratification of persons from high risk groups according to the differences in their health status and the stages of the immune system dysfunction, which might aid a decision of who should receive which alternative vaccination approach [24].

\section{Optimization Challenges in Influenza Vaccination Campaigns}

As mentioned above, current influenza vaccination policy is primarily self-oriented and only indirectly population-oriented. There is no a word, in nowadays vaccination guidelines, on the extent to which the population are to be vaccinated to stop the epidemic growing. Is it possible to estimate the herd immunity threshold, at the beginning, or during the course of the epidemic, and according to that estimation directing further recommendations for vaccine uptake, taking care at the same time on the minimum percentage of subjects from high risk groups need to be protected and the total costs [41]? Are there connections between establishing a real-time herd immunity in the local community and the collective preexisting immunity, gained by the past exposures to influenza viruses? What number of vaccine doses is to be ordered, in order to meet some objective criteria? These and other similar questions should be considered by the public health institutes (PHIs) in the future.

To ensuring success of influenza vaccination, communication campaigns on the benefits of vaccination might be as important as technology advances in vaccine production [42]. Communication strategies may include interpersonal provider-patient relationships and initiatives to incourage target groups (elderly, patients with chronic medical conditions, their families), or to increase awareness of the global community on the benefits of vaccination. Also important is action plan harmonization, the strategy which in many aspects may acquire the characteristics of the social marketing [43]. For these purposes, governments and health authorities need to join the efforts to fairly inform the public, by providing them with accurate, although well-balanced messages. That means that they should be aware of the future consequences of their current activities. Otherwise, communication campaigns may turn towards undesirable directions, by disseminating misinformation, fear and rejection [44]. An important prerequisite, to conducting efficient social campaigns, is to understanding the target population's attitudes, behaviours and concerns $[45,46]$.

\section{Possible Strategies to Improving Vaccination Coverage}

Evidence shows that the best way to alleviate deleterious effects of influenza epidemics, is through effectively designed vaccination programs [47]. As the minimum content, program should include media campaigns, education and financial support of healthcare professionals who deliver vaccines to patients, reminder/recall systems, assessment and feedback of uptake rates and free access to vaccination. Whether it is true, then why, even within the best performed vaccination programs, the vaccination rates frequently maintain low [48]?

Healthcare workers (HCWs), mostly general practitioners (GPs), are the key element of the vaccination programs performance. Not surprisingly then, that $\mathrm{HCWs}$ recommendations is one of the strongest predictors of the vaccine uptake $[46,49,50]$. However, $\mathrm{HCWs}$ are frequently negatively predisposed towards vaccination, be- 
cause of misbeliefs about influenza, its risks and vaccine effectiveness and for reasons such as a lack of knowledge on indications for vaccination, suspicions on the sideeffects and a lack of external support in the form of reminder systems $[48,51,52]$. Therefore, targeting GPs, by means of special programs to educate them and in advance elaborate their activities, might be a strategy to improving vaccination coverage.

Other strategies should address the public widespread attitudes that influenza vaccination is a low priority preventive task $[46,51,53]$. Influenza infection is not directly life threathening and available vaccine is less immunogenic in most of whom is required. So, only clear evidence can increase awareness of the public on the benefits of vaccination. When strong evidence is missing, then fairly, transparently reporting, could aid the decision making. A good example has been provided by the recently published systematic reviews on efficacy and effectiveness of influenza vaccines, showing only modest protection against influenza and its complications in older population [54,55]. However, when older people were stratified according to co-morbid conditions, the impact of influenza was found to be more serious in those at high risk. That means that despite lower immune responsivity, this group is likely to experience higher risk reduction and therefore gain greater benefits after vaccination [56,57]. Thus, although someone can feel influenza vaccination is not necessary, it is still the best intervention available against influenza and its complications.

Another important misperception is on the side-effects. Providing people with on time reports on safety of vaccines, may aid to overcome this barrier [58]. In addition, research on social-cognitive variables has revealed that values such as health beliefs, patterns of behaviours and some cognitive concepts such as "an intention to do", also influence vaccine uptake [46,59]. Better understanding these cognitive processes can help managing patient willingness to accept the vaccine.

An intriguing approach is that vaccination of those who usually disseminate infection, including HCWs and children, might provide protection to persons who are not able to achieve protection directly by vaccination. HCWs who have no willingness to be vaccinated are usually the same ones who in general have negative attitudes towards vaccination [51]. Low rate of vaccination among HCWs in all countries indicate that more attention should be paied on their education [50]. Also, further research, to clearify whether herd immunity, or previous exposures (preexisting antibody titres) are sufficient, or not, to provide protection to HCWs in current epidemics, would be probably helpful. Or studies planned to show whether frequent albeit short-time professional exposures of HCWs are sufficient to allow influenza infection trans- mission, will likely to further add to the evidence.

In this context is also the fact that children are the largest human reservoir of natural influenza infection. A debate pro and con vaccination of children has been conducting for years $[7,9]$. However, apart from the potential benefits this approach may permit, the lifelong effect which priming infection might have on serologic and cell-mediated immune response, should not be forgotten.

\section{Personal Experience}

In my country, Croatia, access to vaccination is free from payment for all persons from high risk groups and HCWs. The majority of doses are delivered by GPs who make an order by counting on past consumptions. The central ordering and distribution point is the regional PHI. Vaccination is variably supported by the media and the PHI information activities. GPs receive recommendations in a written form and are not additionally educated for vaccination. Described situation is similar to many other European countries. The author wanted to get an insight into her own practice, characterized with a large proportion of older and chronically ill patients (Table 1) [60].

An audit analysis of the practice showed that the vaccinated patients were mostly well-being elderly. The structure analysis raised a question on whether the vaccine is delivered to all whom is needed, imposing on GPs a task of more active participation in a decision making (Table 1). The working framework would be within programed vaccination, which would likely to improve the proportion of those vaccinated among patients with particular chronic diseases. This analysis also implies a question of the vaccine supply and the national vaccination policy, according to the recently published study showing that over two-thirds of the world countries did not distribute sufficient doses to cover $10 \%$ of the population and one-third did not reach even $1 \%$ of the population [61]. Patients vaccinated that season were also interviewed by a semi-questionnaire, to gain an insight into repeated vaccinations, motivation for vaccination, a source of information (a doctor, a family,

Table 1. Structure analysis of patients required vaccination in the season $2010 / 11$.

A number of patients $\geq 50$ y $765, \geq 65$ y 486

A number of patients with chronic diseases 744

A total number of patients received a vaccine 210

A number of patients with chronic diseases, vaccinated 111

A proportion of those vaccinated among patients with a particular chronic disease

Diabetes 5/300, Asthma 10/200, COPB 3/56, Chronic heart diseases $45 / 100$

Haemodialysis 3/3, Malignant diseases 45/85 
media, or by yourself) and a pattern of behavior in regard to disease prevention and receiving the protection (Table 2).

As shown in Table 2, vaccinated patients were guided for vaccination by past good experiences and an awareness of the importance of taking a selfprotection. This conclusion, in short, repeats the results of previously published studies which indicate that the past behavior is the best predictor of later adherence to vaccination. As cited by another author, "if behavior is carried out frequently in a stable context, $\cdots$ responses are performed rather automatically" [59]. Similar to these results, other authors also came to the conclusion that "persons who choose to be vaccinated might also be those who are more compliant with medications, exercise regularly, or are more likely to seek medical care early in an illness" [62]. These statements are in line with the prior discussion and clearly indicate a need for programed vaccination.

Since there are no elaborated influenza vaccination protocols, GPs who deliver vaccines to their patients are usually left to make decisions on their own. Information on how GPs manage their activities in this area of preventive medicine is scarce. In a small pilot survey, 23 experienced GPs, specialists, from my region, OsijekBaranja County, were assessed by 36 -item questionnaire on their knowledge and attitudes towards influenza vaccination, including the way they deliver influenza vaccine to their patients and their own behaviour as the target group for influenza vaccination (Table 3) [63].

As shown in Table 3, influenza vaccination is the area of preventive medicine where GPs, in their decisionmaking, largely deal with uncertainties. Experienced doctors tend to develop their own strategies, although there is a need for more sound external support.

\section{Conclusion}

Awareness among health authorities in Europe is increasing that the best way to address current low influenza vaccination rates is to adopt well-elaborated and cost-effective national vaccination programs and to harmonize them throughout European countries. To reach this goal, efforts should focus on the widespread misconceptions on vaccine safety and effectiveness. Only through continuous research, it will be possible to get information we need to make informed decisions on how to handle this matter efficiently. This manuscript has intention to emhasize some of the uncertainties which seek to be resolved. We will need a central authorative body, to continuously create and disseminate recommendations based on using up-to-date evidence. In the local communities, executive protocols should be implemented, specifically elaborated to cope with the characteristics of the local populations and the healthcare
Table 2. Conclusions made upon the analysis of a questionnaire.

The number exceeding 10 in 145/210 (2/3) of vaccinated patients

The main reasons for vaccination

A decline in the health status (20 patients)

An intention to protect grandchildren (2 patients)

A self-protection (all others)

They made a decision for a vaccination mainly by themselves

They were not afraid of side-effects of a vaccine as being guided by positive past experiences

They showed an intention of buying vitamins and supplements and of taking other preventive measures (diet, hand wash, avoiding the crowded places) during the flu season

Table 3. Summary of the results of an interview with family physicians $(\mathrm{N}=23)$ on their knowledge and attitudes on influenza vaccination.

\begin{tabular}{|c|c|}
\hline Item & $\begin{array}{l}\% \text { of interviewed } \\
\text { doctors }\end{array}$ \\
\hline $\begin{array}{l}\text { Possessing an appropriate knowledge on high risk } \\
\text { groups for influenza vaccination and influenza v. } \\
\text { infection related complications }\end{array}$ & $100 \%$ \\
\hline A custom of calling patients for vaccination & $60 \%$ \\
\hline $\begin{array}{l}\text { An average proportion of patients with chronic } \\
\text { diseases vaccinated per family physician team }\end{array}$ & $10 \%-15 \%$ \\
\hline $\begin{array}{l}\text { Physicians satisfied with these results } \\
\text { (the main explanation: most of volunteers are } \\
\text { covered by vaccination) }\end{array}$ & $55 \%$ \\
\hline $\begin{array}{l}\text { Physicians not satisfied with these results } \\
\text { (the main explanation: the achieved vaccination } \\
\text { rates for chronically ill patients are not enough to } \\
\text { include all patients who have need of vaccination) }\end{array}$ & $45 \%$ \\
\hline Vaccination of chronically ill children & $15 \%$ \\
\hline $\begin{array}{l}\text { An assumption about vaccination of } \\
\text { healthy children }\end{array}$ & $15 \%$ \\
\hline $\begin{array}{l}\text { Should be vaccinated } \\
\text { Should not be vaccinated }\end{array}$ & $85 \%$ \\
\hline $\begin{array}{l}\text { Attitudes towards vaccination of patients } \\
\text { with malignant diseases }\end{array}$ & $50 \%$ \\
\hline $\begin{array}{l}\text { Family physicians who systematically } \\
\text { perform vaccination for this patients' group }\end{array}$ & $50 \%$ \\
\hline $\begin{array}{l}\text { Family physicians who assume that this patients' } \\
\text { group should not be vaccinated }\end{array}$ & $50 \%$ \\
\hline Family physicians requiring the flu vaccine & $56 \%$ \\
\hline $\begin{array}{l}\text { Satisfaction with the public health services } \\
\text { in providing them support during } \\
\text { vaccination campaign }\end{array}$ & $60 \%$ \\
\hline $\begin{array}{c}\text { Satisfied } \\
\text { Not satisfied } \\
\text { (An explanation: many key decisions and } \\
\text { procedures, including medical waste disposal, } \\
\text { are left them on their own care) }\end{array}$ & $40 \%$ \\
\hline
\end{tabular}


systems. To fully realize the programed vaccination approach, E-health record would be necessary, to support the sustainable development of this idea, by ensuring data collection for on-going research. Data collected in a standardized way would allow computer-based modeling which, in turn, would intensify epidemiologic research. Only by using this approach, it would be possible to find answers to dubious questions, such as: Does the individual's immune system become overloaded after $15-20$ of consecutive vaccinations; whether this schedule is necessary, or would interval vaccination be a more appropriate approach?

\section{REFERENCES}

[1] T. Szucs, "The Socio-Economic Burden of Influenza," Journal of Antimicrobial Chemotherapy, Vol. 44, No. 1, 1999, pp. 11-15. doi:10.1093/jac/44.suppl_2.11

[2] W. W. Thompson, D. K. Shay and E .Weintraub, "Influenza-Associated Hospitalization in the United States," Japan Automobile Manufacturers Association, Vol. 292, No. 11, 2004, pp. 1333-1340. doi:10.1001/jama.292.11.1333

[3] R. G. Webster, "Immunity to Influenza in the Elderly," Vaccine, Vol. 18, No. 16, 2000, pp. 1686-1689. doi:10.1016/S0264-410X(99)00507-1

[4] J. E. McElhaney and E. B. Effros, "Immunosenescence: What Does It Mean to Health Outcomes in Older Adults?" Current Opinion in Immunology, Vol. 21, No. 4, 2009, pp. 418-424. doi:10.1016/i.coi.2009.05.023

[5] W. E. P. Beyer, I. A. de Brujin, A. M. Palache, R. G. J. Westendorp and A. D. M. E. Osterhaus, "Protection against Influenza after Annually Repeated Vaccination. A Meta-Analysis of Serologic and Field Studies," Archives of Internal Medicine, Vol. 159, No. 2, 1999, pp. 182-188. doi:10.1001/archinte.159.2.182

[6] L. B. Brydak and M. Machala, "Humoral Immune Response to Influenza Vaccination in Patients from High Risk Groups,” Drugs, Vol. 60, No. 1, 2000, pp. 35-53. doi:10.2165/00003495-200060010-00004

[7] Center for Disease Control and Prevention, Immunization Practices Advisory Committee, "Prevention and Control of Influenza. Recommendations of the Advisory Comittee on Immunization Practices," Morbidity, Mortality Weekly Report, Vol. 56, No. RR-6, 2007, pp. 1-54.

[8] P. O. Lang, D. Samaras, N. Samaras, S. Govind and R. Aspinall, "Influenza Vaccination in the Face of Immune Exhaustion: Is Herd Immunity Effective for Protecting the Elderly?" Influenza Research and Treatment, Vol. 2011, 2011, Article ID 419216. doi:10.1155/2011/419216

[9] F. M. Munoz, "The Impact of Influenza in Children," Seminars in Pediatric Infectious Diseases, Vol. 13, No. 2, 2002, pp. 72-78. doi:10.1053/spid.2002.122992

[10] K. M. Harris, J. Maurer and A. L. Kellerman, "Influenza Vaccine-Safe, Effective and Mistrusted," The New England Journal of Medicine, Vol. 363, No. 23, 2010, pp. 2183-2185. doi:10.1056/NEJMp1012333
[11] K. L. Nicholl, "Improving Influenza Vaccination Rates among Adults," Cleveland Clinic Journal of Medicine, Vol. 73, No. 11, 2006, pp. 1009-1015. doi:10.3949/ccjm.73.11.1009

[12] Council of the European Union, "Council Recommedation of 22 December 2009 on Seasonal Influenza Vaccination," Official Journal of the European Union, Vol. L384, No. 1, 2009, pp. 71-72.

[13] J. P. Michel, C. Chidiac and B. Grubeck-Loebenstein, "Coalition of Advocates to Vaccinate of Western European Citizens Aged 60 Years and Older," Aging Clinical and Experimental Research, Vol. 21, No. 3, 2009, pp. 254-257.

[14] Th. M. E. Govaert, M. W. J. Sprenger, G. J. Dinant, K Aretz, N. Masurel and J. A. Knottnerus, "Immune Response to Influenza Vaccination of Elderly People. A Randomized Double-Blind Placebo-Controlled Trial," Vaccine, Vol. 12, No. 13, 1994, pp. 1185-1189. doi:10.1016/0264-410X(94)90241-0

[15] A. M. Palache, I. A. de Bruijn and J. Nauta, "Influenza Immunisation. I. Influenza Vaccination Policies and New Vaccine Developments. II. Fifteen Years's Experience with a Subunit Influenza Vaccine," Journal Clinical Research, Vol. 2, 1999, pp. 111-139.

[16] C. Van Hoecke, V. Prikazsky, I. Uto and C. Menschikowski, "Immunogenecity of an Inactivated Split Influenza Vaccine in Institutionalized Elderly Patients," Gerontology, Vol. 42, No. 4, 1996, pp. 190-198. doi:10.1159/000213792

[17] S. Allsup, A. Haycox, M. Regan and M. Gosney, "Is Influenza Vaccination Cost Effective for Healthy People between Ages 65 and 74 Years? A Randomized Controlled Trial," Vaccine, Vol. 23, No. 5, 2004, pp. 639-645.

[18] I. A. De Brujin, E. J. Remarque, C. M. Jol-van der Zijde, M. J. D. van Tol, R. G. J. Westendorp and D. L. Knook, "Quality and Quantity of the Humoral Immune Response in Healthy Elderly and Young Subjects after Annually Repeated Influenza Vaccination," The Journal of Infectious Diseases, Vol. 179, No 1, 1999, pp. 31-36. doi: $10.1086 / 314540$

[19] K. L. Nichol, J. D. Nordin and D. B. Nelson, "Effectiveness of Influenza Vaccine in the Community-Dwelling Elderly," The New England Journal of Medicine, Vol. 357, No. 26, 2007, pp. 1373-1381. doi:10.1056/NEJMoa070844

[20] W. E. Beyer, A. M. Palache M. J. Sprenger, E. Hendriksen, J. J. Tukker, R. Darioli, G. L. van der Water, N. Masurel and A. D. Osterhaus, "Effects of Repeated Annual Influenza Vaccination on Vaccine Sero-Response in Young and Elderly Adults," Vaccine, Vol. 14, No. 14, 1996, pp. 1331-1339. doi:10.1016/S0264-410X(96)00058-8

[21] M. Hara, K. Tanaka and Y. Hirota, "Immune Response to Influenza Vaccine in Healthy Adults and the Elderly. Association with Nutritional Status," Vaccine, Vol. 23, No. 12, 2005, pp. 1457-1463.

[22] L. Majnarić-Trtica, M. Zekić-Sušac, N. Šarlija and B. Vitale, "Prediction of Influenza Vaccination Outcome by Neural Networks and Logistic Regression," Journal of Biomedical Informatics, Vol. 43, No. 5, 2010, pp. 774-781. 
doi:10.1016/j.jbi.2010.04.011

[23] L. Trtica-Majnarić, N. Sarlija and B. Vitale, "Modelling Influenza Vaccination Outcomes," World Journal Vaccines, Vol. 2, No. 1, 2012, pp. 12-20.

doi:10.4236/wjv.2012.21002

[24] L. Majnarić-Trtica and B. Vitale, "Systems Biology as a Conceptual Framework for Research in Family Medicine: Use in Predicting Response to Influenza Vaccination," Primary Health Care Research \& Development, Vol. 12, No. 4, 2011, pp. 310-321. doi: $10.1017 /$ S1463423611000089

[25] J. C. De Jong, W. E. P. Beyer, A. M. Palache, G. F. Rimmelzwaan and A. D. M. E. Osterhaus, "Mismatch between the 1997/1998 Influenza Vaccine and the Major Epidemic A(H3N2) Virus Strain as the Cause of an Inadequate Vaccine-Induced Antibody Response to This Strain in the Elderly," Journal of Medical Virology, Vol. 61, No. 1, 2000, pp. 94-99. doi:10.1002/(SICI)1096-9071(200005)61:1<94::AID-JM V15>3.0.CO;2-C

[26] K. M. Neuzil, "Pandemic Influenza Vaccine Policy-Considering the Early Evidence," The New England Journal of Medicine, Vol. 361, No. 25, 2009, pp. 2424-2435. doi:10.1056/NEJMe0908224

[27] J. E. McElhney, G. S. Meneilly, K. E. Lechelt, B. L. Beattie and R. C. Bleackley, "Antibody Response to Whole-Virus and Split-Virus Influenza Vaccines in Successful Aging," Vaccine, Vol. 11, No. 10, 1993, pp. 10551060. doi:10.1016/0264-410X(93)90133-I

[28] S. A. Plotkin, "Immunologic Correlates of Protection Induced by Vaccination," The Pediatric Infectious Disease Journal, Vol. 20, No. 1, 2001, pp. 63-75. doi:10.1097/00006454-200101000-00013

[29] C. Hannoun, F. Megas and J. Piercy, "Immunogenicity and Protective Efficacy of Influenza Vaccination," Virus Research, Vol. 103, No. 1-2, 2004, pp. 133-138. doi:10.1016/j.virusres.2004.02.025

[30] L. Qin, P. B. Gilbert, L. Corey, M. J. McElrath and S. G. Self, "A Framework for Assessing Immunological Correlates of Protection in Vaccine Trials," The Journal of Infectious Diseases, Vol. 196, No. 9, 2007, pp. 1304-1312. doi: $10.1086 / 522428$

[31] P. K. Tosh, R. M. Jacobson and G. A. Poland, "Influenza Vaccines: From Surveillance through Production to Protection," Mayo Clinic Proceedings, Vol. 85, No. 3, 2010, pp. 257-273. doi:10.4065/mcp.2009.0615

[32] J. H. C. M. Kreijtz, R. A. M. Fouchier and G. F. Rimmelzwaan, "Immune Responses to Influenza Virus Infection," Virus Research, Vol. 162, No. 1-2, pp. 19-30. doi:10.1016/j.virusres.2011.09.022

[33] C. Reis e Sousa, "Activation of Dendritic Cells: Translating Innate into Adaptive Immunity," Current Opinion in Immunology, Vol. 16, No. 1, 2004, pp. 21-25. doi:10.1016/j.coi.2003.11.007

[34] J. E. McElhaney, D. Xie, W. D. Hager, M. B. Barry, Y. Wang and A. Kleppinger, "T Cell Responses Are Better Correlates of Vaccine Protection in the Elderly," The Journal of Immunology, Vol. 176, No. 10, 2006, pp. 6333-6339.
[35] G. F. Rimmelzwaan and J. E. McElhaney, "Correlates of Protection: Novel Generations of Influenza Vaccines," Vaccine, Vol. 26S, No. 4, 2008, pp. D41-D44. doi:10.1016/j.vaccine.2008.07.043

[36] R. D. Kovaiou, D. Herndler-Brandstetter and B. GrubeckLoebenstein, "Age-Related Changes in Immunity: Implications for Vaccination in the Elderly," Expert Reviews in Molecular Medicine, Vol. 9, No. 3, 2007, pp. 1-10. doi:10.1017/S1462399407000221

[37] W. H. Chen, B. F. Kozlovsky, R. B. Effros, B. GrubeckLoebenstein, R. Edelman and M. B. Sztein, "Vaccination in the Elderly: An Immunological Perspective," Trends in Immunology, Vol. 30, No. 7, 2009, pp. 351-359. doi:10.1016/j.it.2009.05.002

[38] H. Kitano, "Systems Biology: A Brief Overview," Science, Vol. 295, No. 5560, 2002, pp. 1662-1664. doi:10.1126/science.1069492

[39] S. Fukuyama and Y. Kawaoka, "The Pathogenesis of Influenza Virus Infections: The Contributions of Virus and Host Factors," Current Opinion in Immunology, Vol. 23, No. 4, 2011, pp. 481-486. doi:10.1016/j.coi.2011.07.016

[40] L. C. Lambert and A. S. Fauci, "Influenza Vaccines for the Future," The New England Journal of Medicine, Vol. 363, No. 21, 2010, pp. 2036-2044. doi:10.1056/NEJMra1002842

[41] E. Shim, L. A. Meyers and A. P. Galvani, "Optimal H1N1 Vaccination Strategies Based on Self-Interest versus Group Interest," BMC Public Health, Vol. 11, No. S1, 2011, p. S4. doi:10.1186/1471-2458-11-S1-S4

[42] K. M. Harris, J. Maurer and A. L. Kellermann, "Influenza Vaccine-Safe, Effective and Mistrusted," The New England Journal of Medicine, Vol. 363, No. 23, 2010, pp. 2183-2185.

[43] S. C. Jones and D. Iverson, "Pandemic Influenza: A Global Challenge for Social Marketing," Health, Vol. 4, No. 10A, 2012, pp. 955-962. doi:10.4236/health.2012.430146

[44] S. C. Jones, L. Waters, F. Byrne, D. Iverson, M. Sutherland, J. Gold and C. Puplick, “ 'Body Bags Ready': Print Media Coverage of Avian Influenza in Australia," Health, Vol. 4, No. 10A, 2012, pp. 927-932. doi: $10.4236 /$ health.2012.430142

[45] D. Ofri, "the Emotional Epidemiology of H1N1 Influenza Vaccination," The New England Journal of Medicine, Vol. 361, No. 27, 2009, pp. 2594-2595.

[46] K. L. Nichol, R. Mac Donald and M. Hauge, "Factors Associated with Influenza and Pneumococcal Vaccination Behaviour among High-Risk Adults," Journal of General Internal Medicine, Vol. 11, No. 11, 1996, pp. 637-677. doi:10.1007/BF02600158

[47] A. G. Jansen, E. A. Sanders, K. L. Nichol, A. M. van Loon, A. W. Hoes and E. Hak, "Decline in InfluenzaAssociated Mortality among Dutch Elderly Following the Introduction of a Nationwide Vaccination Program," Vaccine, Vol. 26, No. 44, 2008, pp. 5567-5574. doi:10.1016/j.vaccine.2008.08.003

[48] F. Hofmann, C. Ferracin, G. Marsh and R. Dumas, "In- 
fluenza Vaccination of Healthcare Workers: A Literature Review of Attitudes and Beliefs," Infection, Vol. 34, No. 3, 2006, pp. 142-147. doi:10.1007/s15010-006-5109-5

[49] D. R. Johnson, K. L. Nichol and K. Lipczynski, "Barriers to Adult Immunization," The American Journal of Medicine, Vol. 121, No. S7, 2008, pp. S28-S35.

[50] J. P. Michel, P. O. Lang and J. P. Bayens, "Flu Vaccination Policy in Old Patients: Need for Harmonization and National Public Health Recommendations throughout Europe," Vaccine, Vol. 27, No. 2, 2009, pp. 182-183. doi:10.1016/j.vaccine.2008.10.072

[51] J. P. Bayens, "Ensuring the Willingness to Vaccinate and Be Vaccinated," Expert Review of Vaccines, Vol. 9, No. S3, 2010, pp. 11-14. doi:10.1586/erv.10.28

[52] K. L. Nichol and R. Zimmerman, "Generalist and Subspecialist Physicians' Knowledge, Attitudes and Practices regarding Influenza and Pneumococcal Vaccinations for Elderly and Other High-Risk Patients," Archives of Internal Medicine, Vol. 161, No. 22, 2001, pp. 2702-2708. doi:10.1001/archinte.161.22.2702

[53] L. Ward and J. Draper, "A Review of the Factors Involved in Older People's Decision Making with Regard to Influenza Vaccination: A Literature Review," Journal of Clinical Nursing, Vol. 17, No. 1, 2008, pp. 5-16.

[54] T. Jefferson, D. Rivetti, M. Rudin, C. Di Pietrantonj and V. Demicheli, "Efficacy and Effectiveness of Influenza Vaccines in Elderly People: A Systematic Review," Lancet, Vol. 366, No. 9492, 2005, pp. 1165-1174. doi:10.1016/S0140-6736(05)67339-4

[55] M. T. Osterholm, N. S. Kelley, A. Sommer and E. A. Belongia, "Efficacy and Effectiveness of Influenza Vaccines: A Systematic Review and Meta-Analysis," The Lancet Infectious Diseases, Vol. 12, No. 1, 2012, pp. 3644.

[56] E. Hak, J. Nordin, F. Wei, J. Mullooly, S. Poblete, R. Strikas and K. L. Nichol, "Influence of High-Risk Medi- cal Conditions on the Effectiveness of Influenza Vaccination among Elderly Members of 3 Large Managed-Care Organizations," Clinical Infectious Diseases, Vol. 35, No. 4, 2002, pp. 370-377. doi:10.1086/341403

[57] P. O. Lang, A. Mendes, J. Socquet, N. Assir, S. Govind and R. Aspinall, "Effectiveness of Influenza Vaccine in Aging and Older Adults: Comprehensive Analysis of the Evidence," Clinical Interventions in Aging, Vol. 7, 2012, pp. 55-64.

[58] F. C. Zhu, H. Wang, H. H. Fang, J. G. Yang, X. J. Lin and X. F. Liang, "A Novel Influenza A (H1N1) Vaccine in Various Age Groups," The New England Journal of Medicine, Vol. 361, No. 25, 2009, pp. 2414-2423. doi:10.1086/341403

[59] A. Ernsting, S. Lippke, R. Schwarzer and M. Schneider, "Who Participates in Seasonal Influenza Vaccination? Past Behavior Moderates the Prediction of Adherence," Advances in Preventive Medicine, Vol. 2011, 2011, Article ID: 148934. doi:10.4061/2011/148934

[60] B. Bulat and L. Majnaric-Trtica, "Learning More about Older Patients Vaccinated against Influenza Can Direct Future Vaccination Strategy Planning," The Book of Abstracts of the 17th WONCA Europe Conference, Warsaw, 8-11 September 2011, p. 205.

[61] A. Palache, "Seasonal Influenza Vaccine Provision in 157 Countries (2004-2009) and the Potential Influence of National Public Health Policies," Vaccine, Vol. 29, No. 51, 2011, pp. 9459-9466. doi:10.1016/j.vaccine.2011.10.030

[62] J. D. Treanor, "Influenza-The Goal of Control," The New England Journal of Medicine, Vol. 357, No. 14, 2007, pp. 1439-1441. doi:10.1056/NEJMe078140

[63] L. Kramar, P. Vojnovic, M. Marincic and L. T. Majnaric, "Knowledge and Attitudes of Family Physicians about Influenza Vaccination," The Book of Abstracts of the 18th WONCA Europe Conference, Vienna, 4-7 July 2012, p. 269. 